Relations industrielles

Industrial Relations

\title{
Les camps de travaux forcés en Tchécoslovaquie
}

\section{François Bregha}

Volume 6, numéro 1, décembre 1950

URI : https://id.erudit.org/iderudit/1023245ar

DOI : https://doi.org/10.7202/1023245ar

Aller au sommaire du numéro

Éditeur(s)

Département des relations industrielles de l’Université Laval

ISSN

0034-379X (imprimé)

1703-8138 (numérique)

Découvrir la revue

Citer cet article

Bregha, F. (1950). Les camps de travaux forcés en Tchécoslovaquie. Relations industrielles / Industrial Relations, 6(1), 4-11. https://doi.org/10.7202/1023245ar

Tous droits réservés (C) Département des relations industrielles de l’Université Laval, 1950
Ce document est protégé par la loi sur le droit d'auteur. L’utilisation des services d'Érudit (y compris la reproduction) est assujettie à sa politique d'utilisation que vous pouvez consulter en ligne.

https://apropos.erudit.org/fr/usagers/politique-dutilisation/ 


\title{
Les camps de travaux torcés en Tchécoslovaquie
}

\author{
François Bregha, de linstitut tchécoslovaque à l'étranger
}

Cette étude nous donnera les raisons officielles de l'établissement des camps de travaux forcés, tel que le laissent entendre les représentants du régime communiste tchécoslovaque.

L'établissement d'un Etat socialiste - qui assure la subsistance de ses citoyens, qui répartit la production nationale d'après des critères objectifs - tels le besoin et le mérite implique nécessairement la législation de l'obligation de travail, avec des sanctions contre ceux qui la violent. Sans la pression du chômage (qui n'existe pas, évidemment, dans un Etat socialiste), il serait impossible de maintenir la discipline et l'intensité du travail si l'Etat ne possédait pas des moyens de contrainte.

C'est par cette nécessité de sanctions que les régimes communistes cherchent à justifier l'établissement des Camps de travaux forcés. Selon la propagande communiste, les CTF* sont des centres d'éducation ou de rééducation pour ceux qui fuient le travail, qui ne produisent rien pour le bien commun de la société et qui vivent ainsi à ses dépens. Selon la propagande communiste, les CTF en URSS qui servent de modèles aux pays satellites, sont des lieux de travail joyeux, que les rééduqués refusent même de quitter; ils ne subissent aucune contrainte sauf celle de l'obligation de travailler comme tout autre citoyen et ouvrier, dans des conditions favorables et agréables, en recevant le même salaire que les ouvriers libres. L'éducation politique leur démontre l'immoralité de leur attitude passée, et s'ils la reconnaissent et prouvent leur changement d'opinion par un dévouement réel au travail, ils peuvent même obtenir

(*) Dans tout le reste du texte, nous emploierons ces trois lettres pour désigner les « camps de travaux forcés ». des distinctions et ètre libérés tout de suite. On n'oublie jamais de citer les cas dans lesquels le grand Staline lui-même attribue aux détenus des CTF le titre de «héros du travail ».

Les rééduqués reviennent des CTF comme des «hommes nouveaux» transformés en partisans du travail et en bâtisseurs ardents de la société nouvelle et juste, de la société socialiste. Pour des raisons de propagande, il est strictement défendu, en Tchécoslovaquie, d'appeler les camps autrement que «Camps de travaux forcés 》 et leurs habitants « rééduqués ».

Les chapitres suivants résument les informations rassemblées par les organisations de résistance, formant le « Parti du Travail », lequel est considéré complètement illégal en Tchécoslovaquie. Les informations ont été rassemblées dans les CTF, dans les Commissions d'envoi aux CTF, auprès des parents et amis des personnes soumises au séjour dans les dits camps.

Ils montrent nettement que les CTF ne sont point une institution ayant pour but l'éducation ou la punition de ceux qui fuient le travail, portant ainsi préjudice à la société et violant le devoir de travail établi par la Constitution de la République; ils montrent qu'au contraire, les CTF ne sont que l'instrument de l'oppression politique et qu'ils ont été établis consciemment dans ce but même.

\section{1) Base légale}

La première atteinte portée au principe du libre choix de la profession a été ocçasionnée par les circonstances exceptionnelles dans lesquelles se trouvait la Tchécoslovaquie après sa libération en 1945 . Elle résulte du Décret no 32 du Président de la République qui avait pour but

La Revue des relations industrielles 
de faciliter la tâche des autorités d'Etat, au moment où il était absolument nécessaire de répartir le travail indispensable entre les citoyens, même contre leur gré. Il s'agissait là de travaux d'intérêt commun, surtout en ce qui concerne la reconstruction du pays. C'est dans ce sens qu'était appliquée cette loi. Elle permettait d'obliger les hommes entre 18 et 55 ans et les femmes entre 18 et 45 ans, à effectuer un travail autre que celui de leur profession, pendant une durée d'un an et en cas d'extrême nécessité, pendant 18 mois.

Certains individus étaient cependant dispensés de cette obligation: les personnes faisant leur service militaire; les étudiants et autres personnes suivant régulièrement des cours; les femmes enceintes à partir du 5ème mois de grossesse; les femmes ayant à leur charge au moins un enfant, etc.

Une autre source de limitation de la liberté de travail réside dans la Constitution du 9 mai 1948 (établie déjà après le coup d'Etat) qui stipule, dans le passage concernant les « devoirs fondamentaux des citoyens envers l'Etat et la société » (paragraphe 32): «Tout citoyen est obligé de travailler selon ses capacités et de contribuer, par son travail, au bien commun. »

Ni le Décret du Président de la République, ni la Constitution du 9 mai ne donnaient cependant aux organismes d'Etat la liberté de mettre cela en pratique sous forme de persécution politique des adversaires. C'est seulement la Loi concernant les Camps de Travaux Forcés, du 25 octobre 1948 qui rend possible une telle atteinte à la liberté individuelle. C'est par elle que le Parti communiste couvre son action d'un voile de légalité. La loi nouvelle est une suite logique de la Loi pour la défense de la République démocratique populaire. Toutes deux poursuivent le même but: opprimer les ennemis du régime communiste à l'intérieur de l'Etat.
- Le paragraphe 1 de la dite loi institue l'établissement de Camps de travaux forcés pour des raisons d'\& Education des citoyens au travail comme au devoir de citoyen et l' Utilisation de leurs capacités de travail pour le bien commun. »

- Le paragraphe 2 dit: Peuvent être contraintes aux travaux forcés les personnes âgées de 18 à 60 ans: a) qui évitent le travail ou menacent l'établissement de l'ordre de la démocratie populaire ou de sa vie économique, ainsi que les personnes qui -les y aident; b) les personnes condamnées pour l'un des actes prévus par la Loi pour la Défense de la République et la Loi contre le Marché noir.

- Le paragraphe 3 fixe la durée des travaux forcés de 3 mois à 2 ans.

- Le paragraphe 4 prévoit de plus les mesures suivantes contre les condamnés: a) confiscation du logement; b) administration nationale de l'entreprise ou de la propriété privée de la personne condamnée; c) révocation de l'autorisation d'exercice de la profession.

Il est évident que cette loi vise surtout les paysans, les artisans et les petits entrepreneurs, mais le paragraphe 2 offre également la possibilité de persécuter tous les citoyens.

- Enfin le paragraphe 4 montre surtout l'arrière-plan de cette loi qui est d'ordre économique.

Voilà la base sur laquelle la justice communiste appuie le droit d'envoyer aux travaux forcés toute personne qui semble suspecte. Le paragraphe le plus important est le deuxième qui prévoit l'envoi aux camps de ceux qui «menacent la démocratie populaire.» Il faut noter que cette «justice» $n$ ' implique pas la nécessité d'accomplissement d'un acte illégal ou de violation concrète d'une loi; elle n'explique pas non plus en quoi consiste l'attitude ou l'action dangereuse pour «la démocratie populaire.» (Remarque: En outre, l'expression «la démocratie populai- 
re » n'est expliquée nulle part par quelque norme juridique. Selon l'explication officielle du Kominform, la démocratie populaire est un cadre dans lequel l'Etat accomplit les fonctions de dictature du prolétariat).

\section{2) Procédé d'envoi aux CTF}

Les travaux forcés ne sont pas une punition, selon l'interprétation des communistes, et c'est pourquoi (sauf pour les personnes condamnées d'après la Loi pour la Défense de la République) l'envoi aux CTF n'est pas décidé par une cour de justice, mais par une commission spéciale, composée de trois membres et siégeant dans le chef-lieu du département. Ces commissions appelées «Commissions pour l'envoi au CTF en vertu de la loi no $247 / 48$ » sont numérotées (la commission de Prague a le no 1, celle de Brno le no 13). Le commissaire de la Sûreté du Conseil National du Département est le président de la Commission. Le deuxième membre est un représentant des ouvriers et le troisième un représentant du Comité National du Département. Tous trois sont obligatoirement communistes.

Les sources principales de dénonciations, formant la base du procès de la dite commission sont les suivantes: a) la Sûreté d'Etat; b) les organisations et les commissions du parti communiste; c) les Comités des usines; d) les commissions des Comités Nationaux.

La condamnation est prononcée en l'absence de l'accusé, avant même qu'il puisse s'en douter. Il est arrêté immédiatement par un membre du Corps de sûreté nationale et transféré d'abord dans un centre de concentration, au chef-lieu de département: à Prague, ce centre est situé dans le camp de concentration Svaty Jan pod Skalou (St-Jean sous le Rocher); à Brno, dans le bâtiment de la prison pour travaux forcés, rue Lidice, où a lieu l'examen médical. Suivant son résultat, les détenus sont classés généralement en deux catégories. La catégorie A est destinée aux mines et est envoyée dans les CTF rattachés aux mines; la catégorie $B$ doit travailler à la surface et est envoyée aux CTF rattachés aux usines. Les femmes sont détenues dans les camps spéciaux.

Le médecin étant toujours celui de la police soumis aux ordres de ses supérieurs, l'examen médical devient souvent une plaisanterie. Il est établi que le commandant du Corps de Sûreté nationale Grig (commandant de tous les CTF de Moravie) attaqua un médecin en l'accusant de reconnaître tous les détenus comme étant aptes pour un travail réduit seulement (catégorie B) et lui ordonna de les envoyer tous à Oslavany (mines). C'est ainsi que fut déclaré apte aux travaux dans les mines un postier mesurant 5'4" et ne pesant que 102 livres, qui avait été jugé inapte pour le service militaire, et qui s'évanouit à sa première descente au fond de la mine.

En général, on observe pourtant la division en deux groupes, comme il est mentionné plus haut. En particulier, les personnes portant des lunettes ne sont pas envovées dans les mines. ${ }^{1}$

D'ailleurs, les médecins communistes veillent eux-mêmes à ce que nul n'échappe à son sort. Le directeur du plus grand hôpital de Brno, l'hôpital Ste-Anne, demanda en novembre 1949 à la direction des CTF en Moravie de ne pas trop respecter les certificats médicaux de l'hôpital qui sont en général trop «indulgents ». ${ }^{2}$

Une fois transférés au CTF, les détenus y reçoivent leur « Mandat d'incorporation au CTF », avec un retard de deux ou trois semaines, ce qui, selon l'oninion des rééduqués entraîne l'impōssibilité de faire l'appel en temps voulu. Le mandat d'incorporation mentionne la durée du

(1) PTT, Memorandum F, concernant létablissement des CTF, pp. 22-23.

(2) PTT, Rapport no 10. 
séjour au CTF, les autres mesures prises contre le détenu (expulsion de la famille, confiscation de ses biens, administration nationale de son entreprise, etc.) et les raisons d'incorporation. Les raisons mentionnées sont: 1) vous avez tenté de vous enfuir à l'étranger; 2) vous aviez conscience des actions punissables sans les dénoncer; 3) vous avez transporté des personnes à l'étranger. Lorsque aucune action punissable ne peut être prouvée, on justifie l'incorporation par les raisons suivantes: 1) vous êtes une personne suspecte du point de vue politique, et susceptible d'entraver la construction de l'ordre démocratiano populaire; 2) vous n'avez pas une attitude positive envers la République.

L'appel contre la mesure d'incorporation doit être envoyé au ministère de l'Intérieur dans un délai de 15 jours, par la voie de la Commission qui a pris la décision. Il n'est remis, en général, au Ministère de l'Intérieur qu'au bout de trois mois. C'est ainsi qu'un citoyen même complètement innocent est forcé de travailler au moins trois mois dans le CTF. L'appel n'est accepté que dans les cas absolument exceptionnels. ${ }^{3}$ Comme il $y$ avait un grand nombre d'interventions, il fut interdit de plaider pour les détenus sous peine d'envoi dans les CTF. Cette interdiction était affichée au Ministère de l'Intérieur.

\section{3) Règlements}

Aucun règlement n'est connu; il est vraisemblable qu'il n'en existe pas. En général, on utilise les règlements des prisons, interprétés selon les idées des gardiens, et assimilés aux pratiques des camps de concentration allemands.

Les détenus sont surveillés soit par les gardiens de prisons, soit par les membres de la Sûreté nationale ou

(3) Au début de novembre 1949, tous les appels ont été refusés par le Ministère de I'Intérieur. - Rapport No 19 du PTT. par la milice ouvrière. Ils peuvent (théoriquement) recevoir des lettres en nombre illimité. Toute correspondance est naturellement censurée. Ils peuvent également recevoir des visites, en présence des gardiens et compte tenu de la catégorie à laquelle ils appartiennent. La lère catégorie est celle des détenus de courte durée. La 2ème catégorie est celle des détenus incorporés pour une longue durée. Et à la 3ème catégorie appartiennent ceux qui ont été condamnés en vertu de la Loi pour la Défense de la République démocratique populaire.

Les détenus de la lère catégorie peuvent recevoir une visite tous les 15 jours, ceux de la 2ème catégorie, une visite toutes les 6 semaines; ceux de la 3ème catégorie, une visite tous les 2 mois.

Dans l' «Action LC》 (Lutte des classes) les détenus ont été classés en 4 groupes $A, B, C, D$. La raison de ce classement n'est pas connue.

L'horaire de travail est inégal; en général, il est de 8 heures par jour. A Vitkovice par exemple la durée de travail est de 6 a.m. à 2 p.m. et de 4 p.m. à 10 p.m., en forme de «brigades volontaires ». Nul n'est obligé de travailler dans la brigade, mais en ne le faisant pas, on s'expose au danger d'être considéré comme «paresseux 》 ou «oisif 》 et de voir se prolonger considérablement la durée du séjour dans le camp, ou d'être déporté dans un autre camp de travail plus pénible: Jachymov (Joachimsthal) et Hodonin surtout.

Au CTF de Jachymov (Joachimsthal), les heures de travail sont fixées de 6 a.m. à 2 p.m. dans les mines, et ensuite 3 heures supplémentaires à la surface. Au CTF Horni Slavkov, les heures de travail sont fixées de 7 a.m. à 3 p.m., plus 3 heures à la surface. (Rapport no $17 \mathrm{du}$ Rassemblement de la Résistance Tchécoslovaque - selon les expériences de l'informateur luimême). 
4) Nombre de camps et situation géographique

Les camps sont situés en des endroits éloignés des routes et chemins de fer; leur situation géographique n'est pas connue. On peut admettre cependant qu'il y a au moins un camp dans chaque arrondissement. Les camps sont assez dissemblables quant à l'étendue, au nombre des détenus et à la nature du travail.

La plupart des camps se trouvent dans les régions houillères et industrielles. Certains camps cependant approvisionnent en main-d'oeuvre différentes entreprises industrielles et agricoles. Les prisonniers politiques plus importants ou plus dangereux pour le régime, sont envoyés presque exclusivement dans les mines de charbon, de kaolin et d'uranium. Les mines de Jachymov (Joachimsthal) sont réputées pour leur travail très dur, le manque d'hygiène et le mauvais aménagement des mines ne donnent aucune sécurité aux ouvriers travaillant dans le soussol.

En Bohême, les camps les plus importants sont ceux de Kladno (région houillère), nord-ouest de Prague, et de Jilove (Bohême centrale). En Moravie les principaux CTF sont les suivants: dans la région houillère au nord-ouest de la Moravie, il y a les camps de Ostrava (plusieurs camps), de Vitkovice et de Trinec. En Moravie centrale, nous pouvons citer les camps de Kuncice, de Oslavany, de Blansko (plusieurs camps), de Hrusov (mines Stachanov) et de Hodonin. Le camp de Znojmo (Znaim) près de la frontière autrichienne, est destiné exclusivement aux femmes. Le seul camp important en Slovaquie est celui d'Orava, près de la frontière polonaise.

Les camps se divisent selon la nature de travail plus ou moins pénible. Le camp de Hodonin principalement est considéré comme l'un des plus durs. Le camp de Leopoldov est utilisé pour les déportations en URSS.
Le groupe de CTF des mines d'uranium des environs de Jachymov a une situation tout à fait particulière. Le centre de répartition des détenus, pour ce groupe, se trouve à Vitmanov où sont amenés les prisonniers politiques de toute la République. La durée moyenne des condamnations est de 8 ans. Ceux qui sont condamnés à un emprisonnement de longue durée sont transportés dans les wagons spéciaux et accompagnés par des gardiens armés, ceux-ci en nombre à peu près égal à celui des prisonniers. De Vitmanov, les détenus sont dirigés vers les autres CTF de cette région, dont les plus connus sont Jachymov (Joachimsthail) et Horni Slavkov.

Le nombre total des personnes emprisonnées ou détenues en Tchécoslovaquie pour les raisons politiques est tenu secret. Pour le département de Brno, il y avait environ 3,000 personnes vers la fin de juin 1949. En fin de septembre de la même année, le nombre dépassait déjà 11,000 personnes. Le Rapport no 14 du Rassemblement de la Résistance Tchécoslovaque de fin octobre évalue le nombre total, selon une source non-officielle, à 700,000 soit de 5 à $6 \%$ de la population totale de la Tchécoslovaquie. - Le Gouvernement s'efforce de dissimuler ce terrible fait par la dispersion des camps et par la terreur qui empêche les personnes relâchées de faire des récits.

\section{5) Motifs de déportation}

Les motifs de déportation sont très divers, ainsi que cela ressort des âges très différents des déportés (il y a même parmi ceux-ci des jeunes gens et des jeunes filles de 18 ans).

En fait, le procédé est le suivant: un organe quelconque du Parti communiste ou de la Sûreté d'Etat (organisation locale du Parti, ou Conseil de l'usine, ou bureau de la Sûreté d'Etat, ou Commission quelconque du Conseil National Local, etc.) désigne quelqu'un comme étant dou- 
teux ou dangereux; on trouve alors une raison ou plutôt un prétexte pour l'arrêter.

Il y a eu des arrestations à Brno, motivées par les démonstrations du 28 mai 1949, à l'occasion de l'anniversaire de la naissance du président Bénès. La simple présence dans les environs du monument du président Masaryk suffisait pour être arrêté par le Corps de Sûreté Nationale. Pendant l'enquête qui s'ensuivit, la police s'efforça de trouver quelque tache dans le passé des personnes arrêtées. L'opinion de la Commission des $\mathrm{Ca}$ dres du Parti communiste suffit pour l'envoi du détenu dans un CTF.

Une cause d'incorporation dans les CTF qui se retrouve souvent est la propagande anticommuniste sous toutes les formes. On a déporté des lycéens de Brno et de Prostejov dans les CTF, pour avoir distribué des tracts anticommunistes (ce qui n'est, bien entendu, défendu par aucune loi, la Constitution de la République garantissant au contraire la liberté d'expression et d'opinion, etc.) Un autre motif est le refus de faire fusionner sa propre entreprise avec celle d'Etat. Les ouvriers, absents des usines ou des mines pendant 3 jours sans motifs suffisants, ou se tenant systématiquement à l'écart des brigades volontaires, sont incorporés dans les CTF. Les personnes faisant l'objet d'une enquête de la Cour d'Etat (organisme s'occupant spécialement des crimes politiques) ou de la Sûreté d'Etat et qui devraient être relâchées comme innocentes, sont presque toujours envoyées aux CTF. En outre on détient dans les CTF des voleurs, des prostituées, ce qui aggrave considérablement le sort des détenus politiques.

\section{6) Situation l'intérieur des camps}

Les camps seont entourés par des clôtures de fil de fer barbelé de 10 pieds de hauteur, flanquées de tours, armées de mitrailleuses. Les déte- nus vivent dans des baraques malsaines et humides; il y a souvent plus de vingt hommes dans une chambre faite pour dix. L'hygiène est inexistante. Dans tous les camps connus, il manque de lavabos et de cabinets; les premiers sont remplacés par des auges en bois; les seconds par des trous dans la terre. Au surplus, il manque d'eau. Le service médical fait défaut -.osque partout, même dans les camps où les détenus sont destinés aux travaux dangereux.

La nourriture diffère beaucoup d'un camp à l'autre et laisse d'autant plus à désirer que le camp est mieux surveillé et qu'il dépend plus directement de l'autorité de l'Etat. Dans certains petits camps, les équipes des détenus sont disséminées et les différentes entreprises industrielles ou agricoles dans lesquelles ils travaillent, sont chargées elles-mêmes d'assurer leur subsistance. Dans ce cas, la situation est supportable; mais elle est particulièrement réservée aux prisonniers de droit commun et non aux détenus politiques.

La situation est bien différente dans les camps destinés aux détenus de IIème et IIIIème classe, qui n'ont aucune liberté de mouvement, et dépendent directement du camp où ils sont également nourris (camps d'Hodonin, de Jachymov, de Znojmo, d'Orava, etc.). Dans chacun de ces camps, la nourriture n'est pas suffisante pour entretenir les forces physiques des détenus, ni proportionnée aux travaux exténuants auxquels ils sont astreints. En général, la nourriture consiste en potage, pain noir, pommes de terre; il manque surtout de viande et de graisse.

La question du salaire est également un point faible. Les détenus de Kladno, par exemple, «gagnent $1.50 \mathrm{Kcs}$ par jour (trois cents canadiens selon le cours légal), cependant que l'administration des mines paie pour chacun des travailleurs forcés, à l'administration des CTF $150 \mathrm{Kcs}$ (\$3.00) par jour. Celle-ci prétend devoir retenir cet argent pour 
«payer» le logement et la nourriture des détenus, et pour assurer la subsistance de leurs familles. En réalité, aucune famille de détenus n'a reçu de subsides de l'administration des camps; ces familles sont, au contraire, obligées d'acheter et d'envoyer aux détenus des colis de vivres pour les maintenir en vie. Au surplus, il s'agit souvent de familles dont tous les biens ont été confisqués par l'Etat en même temps que le chef de famille emprisonné. Le salaire des détenus revient ainsi presque totalement au Gouvernement.

L'《éducation politique » est un des éléments les plus importants de la vie des détenus. Chaque détenu doit faire preuve de connaissances de l'idéologie marxiste et des principes de la vie d'une société socialiste, s'il ne veut pas voir se prolonger la durée de sa «rééducation» dans le camp. L'éducation politique dans le sens communiste signifie un changement, une transformation politique de ceux qui acceptent cette transformation et une élimination progressive de ceux qui ne l'acceptent pas. Voilà l'un des principaux buts de cette institution.

Pour se faire une idée de ce qu'est une éducation communiste, il faut remarquer qu'un des meilleurs moyens de prouver son attachement au communisme consiste dans la dénonciation de ceux qui sont moins empressés à se laisser éduquer. Les détenus sont encouragés par leurs - instructeurs » (jeunes officiers de la Sûreté d'Etat) à dénoncer tout réactionnaire. La dénonciation étant le plus sûr moyen pour être relâché, elle est répandue dans certains camps. In faut y ajouter les nombreux agents provocateurs, émissaires de la Sûreté d'Etat.

Les détenus qui reçoivent un avis d'incorporation dans un CTF pour une période supérieure à trois mois, s'efforcent en général pendant les trois premiers mois, de prouver leur loyauté envers le régime par tout moyen possible, pour être libérés, c'est-à-dire pour que leur appel soit accepté. Ayant échoué - le plus souvent - ils tombent dans un état de résignation ou de désespoir qui se manifeste surtout par l'ivresse partout où les détenus ont accès à l'alcool. On a pu remarquer cette disposition d'esprit surtout dans les premières semaines qui ont suivi l'établissement des CTF, principalement parmi les détenus ayant peu de conscience politique. Par contre les derniers rapports des CTF les plus durs mentionnent un esprit remarquable parmi les détenus.

Le traitement des détenus par les gardiens diffère suivant que ceux-ci appartiennent ou non au Parti communiste. Les gardiens communistes, et surtout les officiers qui dirigent l'« éducation politique » aiment à humilier les détenus en les injuriant. Les détenus ne sont pas battus (comme c'était le cas, en règle générale, dans les camps allemands), mais cela se produit parfois. Il n'y a ni moyen légal ni possibilité pratique contre les mauvais traitements.

Par contre, les membres de la Sûreté nationale non-communistes, envoyés en général comme gardiens des CTF par mesure de punition politique, ainsi que les ouvriers libres qui travaillent avec les détenus dans les mines et dans les usines, les aident à supporter leur sort en leur donnant à manger, en leur facilitant le travail, et même en les aidant à fuir. C'est pourquoi les évasions des CTF étaient nombreuses dans certains camps; par exemple, au camp de Vitkovice, il y avait 2 ou 3 prisonniers par jour qui fuyaient.

Depuis quelque temps, on peut observer des cas de disparition de détenus. Il est sûr que certaines de ces disparitions de détenus sont causées par des changements de lieu de travail et par la mobilité des détenus. Mais une autre raison vient de s'ajouter récemment à celle-ci. Selon les rapports des évadés des CTF il devient de plus en plus vraisemblable qu'une certaine proportion des déte- 
nus dans les CTF en Tchécoslovaquie a été envoyée en URSS, et que d'autres attendent le même sort. Le centre de déportation de Tchécoslovaquie en URSS semble être le camp de Leopoldov, dans les Sudètes.

Nous avons exposé ici, en autant que nos renseignements nous le per- mettaient, les conditions qui prévalent dans les camps de travaux forcés de Tchécoslovaquie de même que les raisons officielles de l'établissement de ces camps. Nous nous proposons de donner dans un article subséquent les raisons réelles de l'établissement des camps de travaux forcés.

\section{Etude comparative sur la législation de conciliation et d'arbitrage}

\section{INTRODUCTION}

La présente étude ne constitue pas un exposé des principes qui sont à la base des divers systèmes de règlement des conflits du travail, mais simplement une analyse, une description des principaux types de systèmes de règlement, sans que soient portés des jugements de valeur sur les uns et les autres. Cependant quelques précisions sont nécessaires.

Par «conflits du travail» nous envisageons ici seulement les conflits collectifs et non les conflits individuels, bien que ceux-ci peuvent très souvent se transformer en conflits collectifs. D'ailleurs la distinction entre conflits individuels et conflits collectifs présente plutôt un caractère artificiel comme le font remarquer Rouast et Durand dans leur «Précis de législation industrielle . $^{1}$ Toutefois, s'il n'est pas facile d'établir les critères d'un conflit individuel, il est plus facile de reconnaître les caractéristiques d'un conflit collectif du travail.

(1) Rouast et Durand, Précis de législation industrielle (Droit du Travail), p. 276 et suivantes, Librairie Dalloz, 1948.

\section{Note de la rédaction:}

Sous ce titre nous publierons dans la rovue une série d'articles rédigés par un professeur du Département des relations industrielles de Laval qui à cause de certaines circonstances particulières désire garder l'anonymat.
A ce sujet, Rouast et Durand adoptent la position suivante: «a) Constituent des conflits collectifs, ceux qui mettent en jeu une question de principe, dont la solution intéresse la condition juridique des différents membres d'un groupement. Il en est d'abord ainsi quand le conflit est relatif à la création ou à la revision d'une règle du droit du travail. (Etablissement d'un règlement arbitral des conditions du travail, revision de la convention collective, d'une sentence arbitrale pour cause d'imprévision). De même, présente un caractère collectif le conflit relatif à l'interprétation des règles du droit, qu'il s'agisse des lois, d'usages, de conventions collectives ou de sentences arbitrales. b) Constituent en second lieu des conflits collectifs ceux qui mettent en jeu un intérêt commun à toute ou partie de la collectivité, qui intéressent par exemple la liberté d'opinion, la liberté syndicale, les droits des délégués du personnel, le recours à la grève, même si la solution du différend doit modifier la situation juridique d'un seul membre de l'entreprise. Une mesure individuelle a donc pu donner naissance d un conflit collectif: il suffit de supposer un congédiement, motivé par l'adhésion du salarié à un syndicat. En portant atteinte au droit syndical, l'acte lèse une prérogative de la 